\section{A Discussion on the History of Research in Arithmetic and Reed-Muller Expressions}

Radomir S. Stanković and Tsutomu Sasao

Abstract-This paper discusses early work by Komamiya in Reed-Muller and arithmetic expressions for switching functions.

Index Terms-Adder, arithmetic expression, Boolean expression, EXOR, Reed-Muller expression, Reed-Muller transform.

\section{INTRODUCTION}

A recent discussion [3] shows the origins and earlier work on bitlevel and word-level polynomial expressions for switching and multiple-valued (MV) functions. It was motivated by the study in [17], where the references are restricted mainly to Western research work. These polynomial expressions are related to both spectral representations and differential operators for discrete functions. The coefficients in such representations can be interpreted as some spectral transform coefficients [4] or values of discrete differential or difference operators for discrete functions [5], [19]. In this paper, we provide further information on the earlier work in this area and some alternative points of views.

In this paper, we will discuss the relation between logical expressions and arithmetic expressions. In these expressions, we often use a symbol $x^{a}$. However, the interpretation of this symbol depends on the context. When the symbol $x^{a}$ is used in an logical expression, it represents the literal function

$$
x^{a}= \begin{cases}\bar{x}, & \text { if } a=0 \\ x, & \text { if } a=1 .\end{cases}
$$

However, when $x^{a}$ is used in an arithmetic or in a Reed-Muller expression, it represents the exponentiation function

$$
x^{a}= \begin{cases}1, & \text { if } a=0 \\ x, & \text { if } a=1 .\end{cases}
$$

It is very confusing to use the same symbol to denote two different functions in a paper. Therefore, we will use the symbol $\delta(x-a)$ to represent the literal function and $x^{a}$ to denote the exponentiation function, since we will mainly talking about arithmetic expressions. Note that $\delta(q)$ is the delta function such that $\delta(q)=1$, if $q=0$, and $\delta(q)=0$, if $q \neq 0$.

\section{WORKS BY KOMAMIYA}

Two theorems have been formulated in [11], [15] based on the work of Komamiya (1922-1993) [7]-[9].

Theorem 1 (Arithmetic Expressions): Let $x_{1}, x_{2}, \ldots, x_{n}$ be binary variables and $r$ be an integer defined by $r=x_{1}+x_{2}+\cdots+x_{n}$, where + is the integer addition [7]. Let the binary representation of $r$ be

$$
\left(y_{k}, y_{k-1}, \ldots, y_{1}, y_{0}\right)_{2}, \quad y_{i} \in\{0,1\}, \quad(i=0,1, \ldots, k) .
$$

Manuscript received September 27, 2000. This paper was recommended by Associate Editor M. Papaefthymiou.

R. S. Stanković is with Brace Taskovića 17/29, 18000 Nišs, Yugoslavia (e-mail: rstankovic@bankerinter.net).

T. Sasao is with the Department of Computer Science and Electronics, Kyushu Institute of Technology, Iizuka, 820-8502, Japan (e-mail: sasao@cse.kyutech.ac.jp).

Publisher Item Identifier S 0278-0070(01)06884-1.
In other words

$$
x_{1}+x_{2}+\cdots+x_{n}=2^{k} y_{k}+2^{k-1} y_{k-1}+\cdots+2^{1} y_{1}+y_{0} .
$$

Then

$$
y_{i}=\mathrm{SB}\left(n, 2^{i}\right)
$$

where $\mathrm{SB}(n, k)$ is an $n$-variable symmetric function represented by the EXOR sum of all the products consisting of $k$ positive literals. Also, we define as $\operatorname{SB}(n, 0)=1[12]$

$$
\begin{aligned}
\mathrm{SB}(n, 0) & =1 \\
\mathrm{SB}(n, 1) & =\bigoplus_{i} x_{i} \\
\mathrm{SB}(n, 2)= & \bigoplus_{i<j} x_{i} x_{j} \\
\mathrm{SB}(n, 3)= & \bigoplus_{i<j<k} x_{i} x_{j} x_{k} \\
& \cdots \cdots \\
\mathrm{SB}(n, n)= & x_{1} x_{2} \ldots x_{n} .
\end{aligned}
$$

For example, consider the network that represents the sum of eight inputs as a binary representation. The network has $x_{1}, x_{2}, \ldots, x_{8}$ as inputs and $y_{3}, y_{2}, y_{1}, y_{0}$ as outputs. Then

$$
\begin{aligned}
& y_{3}=\mathrm{SB}(8,8)=x_{1} x_{2} \ldots x_{8} \\
& y_{2}=\mathrm{SB}(8,4)=\bigoplus_{i<j<k<l} x_{i} x_{j} x_{k} x_{l} \\
& y_{1}=\mathrm{SB}(8,2)=\bigoplus_{i<j} x_{i} x_{j} \\
& y_{0}=\mathrm{SB}(8,1)=x_{1} \oplus x_{2} \oplus \cdots \oplus x_{8} .
\end{aligned}
$$

Theorem 2 (Reed-Muller Transform): Let

$$
f(x)=\bigvee_{\boldsymbol{a}} f(\boldsymbol{a}) \delta\left(x_{1}-a_{1}\right) \delta\left(x_{2}-a_{2}\right) \ldots \delta\left(x_{n}-a_{n}\right)
$$

be the minterm expansion of $f$, where $\boldsymbol{x}=\left(x_{1}, x_{2}, \ldots x_{n}\right), \boldsymbol{a}=$ $\left(a_{1}, a_{2}, \ldots a_{n}\right)$ and $a_{i} \in\{0,1\}$. Consider a function

$$
f^{T}(\boldsymbol{x})=\bigoplus_{\boldsymbol{a}} f(\boldsymbol{a}) x_{1}^{a_{1}} x_{2}^{a_{2}} \ldots x_{n}^{a_{n}} .
$$

Then, $\left(f^{T}\right)^{T}=f$.

$f^{T}$ is called the Reed-Muller spectrum of $f$ and the operator $(\cdot)^{T}$ : $f \rightarrow f^{T}$ defined by (3) is called the Reed-Muller transform.

It should be noted that in [15], Theorem 2 is written by using literal functions, while in this paper, it is written by using exponentiation functions. Therefore, they look different. Also, in [11], Nozaki introduced the symbol

$$
\left[\begin{array}{l}
a \\
x
\end{array}\right]
$$

to denote the exponentiation function. 


\section{DISCUSSION}

\section{A. Arithmetic Expressions}

Table I shows relations between operations in logical expressions and arithmetic expressions.

Theorem 1 shows that Komamiya worked with arithmetic expressions of switching functions. A justification for this remark is in the following. In the left-hand side of (1), Komamiya assumed $(0,1)_{C}$ coding of logical variables, while in the right-hand side of (1), he assumed $(0,1)_{\mathrm{GF}(2)}$ coding, where $C$ is the field of complex numbers and GF (2) is the Galois field of order 2. Thus, he implicitly used

$$
(x \wedge y)_{\mathrm{GF}(2)}=(x \cdot y)_{C} .
$$

Similarly, he also used

$$
y \oplus z=y+z-2 y z .
$$

We will consider the case $n=3$ to make it clear. In this case, (1) is written as

$$
x_{1}+x_{2}+x_{3}=2 y_{1}+y_{0}
$$

where

$$
\begin{aligned}
& y_{0}=\mathrm{SB}(3,1)=x_{1} \oplus x_{2} \oplus x_{3} \\
& y_{1}=\mathrm{SB}(3,2)=x_{1} x_{2} \oplus x_{1} x_{3} \oplus x_{2} x_{3} .
\end{aligned}
$$

In this case, (1) is reduced to

$$
x_{1}+x_{2}+x_{3}=2\left(x_{1} x_{2} \oplus x_{1} x_{3} \oplus x_{2} x_{3}\right)+\left(x_{1} \oplus x_{2} \oplus x_{3}\right) .
$$

We use (4) to prove (5). We also use the property $x_{i} x_{i}=x_{i}$, if $x_{i} \in$ $\{0,1\}$ in any of two codings $(0,1)_{\mathrm{GF}(2)}$ and $(0,1)_{C}$. The calculations are given in full details to make the presentation easy to follow

$$
\begin{aligned}
y_{0}= & x_{1} \oplus x_{2} \oplus x_{3} \\
= & \left(x_{1}+x_{2}-2 x_{1} x_{2}\right) \oplus x_{3} \\
= & x_{1}+x_{2}-2 x_{1} x_{2}+x_{3} \\
& -2\left(x_{1}+x_{2}-2 x_{1} x_{2}\right) x_{3} \\
= & x_{1}+x_{2}-2 x_{1} x_{2}+x_{3} \\
& -2 x_{1} x_{3}-2 x_{2} x_{3}+4 x_{1} x_{2} x_{3} . \\
y_{1}= & x_{1} x_{2} \oplus x_{1} x_{3} \oplus x_{2} x_{3} \\
= & \left(x_{1} x_{2}+x_{1} x_{3}-2 x_{1} x_{2} x_{3}\right) \oplus x_{2} x_{3} \\
= & x_{1} x_{2}+x_{1} x_{3}-2 x_{1} x_{2} x_{3}+x_{2} x_{3} \\
& -2\left(x_{1} x_{2}+x_{1} x_{3}-2 x_{1} x_{2} x_{3}\right) x_{2} x_{3} \\
= & x_{1} x_{2}+x_{1} x_{3}-2 x_{1} x_{2} x_{3}+x_{2} x_{3} \\
& -2 x_{1} x_{2} x_{3}-2 x_{1} x_{2} x_{3}+4 x_{1} x_{2} x_{3} \\
= & x_{1} x_{2}+x_{1} x_{3}-2 x_{1} x_{2} x_{3}+x_{2} x_{3} .
\end{aligned}
$$

From these relations, we have

$$
\begin{aligned}
2 y_{1}+y_{0}= & 2\left(x_{1} x_{2}+x_{1} x_{3}-2 x_{1} x_{2} x_{3}+x_{2} x_{3}\right) \\
& +\left(x_{1}+x_{2}-2 x_{1} x_{2}+x_{3}\right. \\
& \left.-2 x_{1} x_{3}-2 x_{2} x_{3}+4 x_{1} x_{2} x_{3}\right) \\
= & 2 x_{1} x_{2}+2 x_{1} x_{3}-4 x_{1} x_{2} x_{3}+2 x_{2} x_{3} \\
& +x_{1}+x_{2}-2 x_{1} x_{2}+x_{3}
\end{aligned}
$$

TABLE I

RELATIONS BETWEEN LOGICAL AND ARITHMETIC EXPRESSIONS

\begin{tabular}{ll}
\hline Logical & arithmetic \\
\hline $\bar{x}$ & $1-x$ \\
$x_{1} \wedge x_{2}$ & $x_{1} x_{2}$ \\
$x_{1} \vee x_{2}$ & $x_{1}+x_{2}-x_{1} x_{2}$ \\
$x_{1} \oplus x_{2}$ & $x_{1}+x_{2}-2 x_{1} x_{2}$ \\
\hline
\end{tabular}

$$
\begin{aligned}
& -2 x_{1} x_{3}-2 x_{2} x_{3}+4 x_{1} x_{2} x_{3} \\
= & x_{1}+x_{2}+x_{3} .
\end{aligned}
$$

Thus, we have verified the correctness of (1) for $n=3$.

It is interesting to note that Aiken's team used arithmetic operations in expressions describing arithmetic circuits of the Harvard Mark 3 and 4 computers [1]. They preferred the factored form rather than the expanded form of arithmetic expressions. Aiken mentioned in the preface of [1] that arithmetic expressions may be useful to design arithmetic circuits. Komamiya used arithmetic expressions to design adders [9]. In October 1952, Komamiya completed a pilot model of a relay computer at a research center of Japanese Government [6].

Theorem 1 is also used to realize symmetric functions by lookup table-type field programmable gate arrays [16]. A system of switching functions, e.g., outputs of an arithmetic circuit, can be represented by a single arithmetic expression instead of a set of logical expressions. In [10], multiple-output functions are efficiently represented by arithmetic expressions. Recently, decision diagrams are used to represent arithmetic expressions compactly. The relationships between decision diagrams and arithmetic expressions are discussed in [20].

\section{B. Reed-Muller Transform}

In Theorem 2, Komamiya converted a logical expression (2) into a Reed-Muller expression (3). Between (2) and (3), he used the relation

$$
f(\boldsymbol{x})=\bigoplus_{\boldsymbol{a}} f(\boldsymbol{a}), \delta\left(x_{1}-a_{1}\right), \delta\left(x_{2}-a_{2}\right), \ldots, \delta\left(x_{n}-a_{n}\right) .
$$

Note that the products

$$
\delta\left(x_{1}-a_{1}\right), \delta\left(x_{2}-a_{2}\right), \ldots, \delta\left(x_{n}-a_{n}\right)
$$

represent the minterm functions and they are pairwise disjoint for different $\mathbf{a}=\left(a_{1}, a_{2}, \ldots, a_{n}\right)$. On the other hand, the products $x_{1}^{a_{1}}, x_{2}^{a_{2}}, \ldots, x_{n}^{a_{n}}$ represent the Reed-Muller functions.

Example 1: For $n=3$, the products $x_{1}^{a_{1}}, x_{2}^{a_{2}}, x_{3}^{a_{3}}$ represent the Reed-Muller functions $\operatorname{rm}(\cdot)$ in the Hadamard ordering defined by

$$
\begin{aligned}
& \operatorname{rm}(0,0,0)=1, \quad \operatorname{rm}(0,0,1)=x_{3}, \\
& \operatorname{rm}(0,1,0)=x_{2}, \quad \operatorname{rm}(0,1,1)=x_{2} x_{3}, \\
& \operatorname{rm}(1,0,0)=x_{1}, \quad \operatorname{rm}(1,0,1)=x_{1} x_{3}, \\
& \operatorname{rm}(1,1,0)=x_{1} x_{2}, \quad \operatorname{rm}(1,1,1)=x_{1} x_{2} x_{3} .
\end{aligned}
$$

Between (2) and (6), Komamiya replaced the literal functions with exponentiation functions to obtain the Reed-Muller transform.

In classical approaches of switching theory, logical expressions are considered as analytic representations of switching functions, where the addition is the logic OR. The Reed-Muller expressions are related to the Boolean ring in which the addition is EXOR. Komamiya discussed the relationship between the coefficients in the Reed-Muller expressions and the binomial coefficients, similarly to other authors attempting to establish a relation between the functions on the real 
field $R$ and the discrete functions [4], [5]. In this case, preserving the basic group structure in terms of EXOR relates to the interpretation of Reed-Muller expressions as analogues of Taylor series expansions and interprets Reed-Muller coefficients as Boolean differences.

The equation $\left(f^{T}\right)^{T}=f$ shows that the Reed-Muller transforms are self-inverse. This can be easily proved in the matrix notation for the Reed-Muller functions by using the properties of the Kronecker product.

\section{Closing REMARKs}

Komamiya's equation

$$
A_{1}+A_{2}+\cdots+A_{n}=d_{m} 2^{m}+d_{m-1} 2^{m-1}+\cdots+d_{1} 2+d_{0}
$$

where $A_{i}, d_{i} \in\{0,1\}$ is fundamental to the design of adders. Various kind of adders can be derived as its special cases. When $n=2$, it corresponds to a half adder and when $n=3$, it corresponds to a full adder.

In addition to the design theory of adders, his design methods involved a new approach to switching theory. The arithmetic and Reed-Muller expressions are particular examples of Fourier series-like expressions over different fields: the complex field $C$ and the Galois field GF(2). Thus, Komamiya was among founders of switching theory as a particular area of applied mathematics.

Komamiya did not publish his work in an English journal, but published it as an internal report. (His paper is 40 pages long and requires considerable effort to understand it.) Therefore, his work was not well known except by Nozaki [11]. From January 1957, he spent a year at the Computation Laboratory, Harvard University, and from September 1962, he stayed at the Digital Computation Laboratory, University of Illinois.

Later, Komamiya noticed Sasao's work [12] and sent him related papers. Unfortunately, Sasao had not enough time to read them at that time. Later, when Sasao was writing a history of switching theory [15], he rediscovered Komamiya's pioneering work. Unfortunately, it was many years after Prof. Komamiya had passed away.

\section{ACKNOWLEDGMENT}

The authors would like to thank Prof. V. D. Malyugin of the Institute of Control, Russian Academy of Science, Moscow, for useful discussions and comments, Prof. J. T. Butler for improving the English presentation, and Prof. Y. Iguchi, who was working with Prof. Komamiya for sending related material.

\section{REFERENCES}

[1] H. H. Aiken, The Annals of the Computation Laboratory of Harvard University, Volume XXVII, Synthesis of Electronic Computing and Control Circuits. Cambridge, MA: Harvard Univ., 1951.

[2] P. L. Butzer and R. S. Stankovíc, Eds., Theory and Applications of Gibbs Derivatives. Beograd, Yugoslavia: Matematički Institut, 1990.

[3] B. J. Falkowski, "A note on the polynomial form of Boolean functions and related topics," IEEE Trans. Comput., vol. 48, pp. 860-864, Aug. 1999.

[4] M. U. Garaev and R. G. Faradzhev, "On an analog of Fourier expressions over Galois fields and its applications to problems of generalized sequential machines," Izv. Akad. Nauk Aizerb. SSR, ser. Fiz.-Techn, i Mat. Nauk, no. 6, pp. 69-75, 1968.

[5] J. E. Gibbs, "Local and Global Views of Differentiation," in Theory and Applications of Gibbs Derivatives, P. L. Butzer and R. S. Stankovíc, Eds. Beograd, Yugoslavia: Matematički Institut, 1990, pp. 1-19.

[6] M. Goto, Y. Komamiya, R. Suekane, M. Takagi, and S. Kuwabara, "Theory and Structure of the Automatic Relay Computer E.T.L. Mark II," Res. Electrotech. Lab., no. 556, Sept. 1956.

[7] Y. Komamiya, "Theory of relay networks for the transformation between the decimal and binary system," Bull. Electrotech. Lab., vol. 15, no. 8, pp. 188-197, Aug. 1951

[8] - "Theory of computing networks," in Proc. 1st Nat. Congr. Applied Mechanics, May 1952, pp. 527-532.

[9] — , "Theory of Computing Networks," in Researches of the Applied Mathematics Section of Electrotechnical Laboratory in Japanese Government Tokyo, Japan, July 10, 1959.

[10] V. D. Malyugin, Paralleled Calculations by Means Arithmetic Polynomials. Moscow, Russia: Russian Academy Sci., 1997. Russian.

[11] A. Nozaki, Switching Theory (in Japanese). Tokyo, Japan: Kyoritsu Shuppan, 1972.

[12] T. Sasao and P. W. Besslich, "On the complexity of MOD-2 sum PLA's," IEEE Trans. Comput., vol. 34, pp. 262-266, Feb. 1990.

[13] T. Sasao and M. Fujita, Eds., Representations of Discrete Functions. Norwell, MA: Kluwer, 1996.

[14] T. Sasao, "Representations of Logic Functions by Using EXOR Operators,", T. Sasao and M. Fujita, Eds. Norwell, MA: Kluwer, 1996, pp. $29-54$.

[15] — Switching Theory for Logic Synthesis. Norwell, MA: Kluwer, 1999.

[16] _ "A new expansion of symmetric functions and their application to nondisjoint functional decompositions for LUT type FPGAs," in Proc. Int. Workshop Logic Synthesis, Dana Point, CA, May 2000, pp. 105-110.

[17] W. G. Schneeweiss, "On the polynomial form of Boolean functions, derivations and applications," IEEE Trans. Comput., vol. 47, pp. 217-221, Feb. 1998.

[18] R. S. Stanković, "Some remarks on Fourier transforms and differential operators for digital functions," in Proc. 22nd Int. Symp. MultipleValued Logic, Sendai, Japan, May 1992, pp. 365-370.

[19] — - "Reed-Muller expressions and Walsh transform," Automatika $i$ Telemekhanika, vol. 57, no. 4, pp. 130-147, 1996. Russian.

[20] R. S. Stanković, T. Sasao, and C. Moraga, "Spectral Transform Decision Diagrams," in Representations of Discrete Functions, T. Sasao and M. Fujita, Eds. Norwell, MA: Kluwer, 1996, pp. 55-92. 